\title{
Image restoration of one-dimensional HgTe crystals formed within walled carbon nanotubes
}

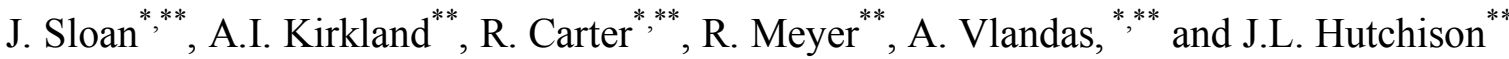 \\ *Inorganic Chemistry, University of Oxford, South Parks Road, OX1 3QR \\ ** Department of Materials, University of Oxford, Parks Road, Oxford, OX1 3PH
}

The insertion and subsequent characterisation of both molecular species and one-dimensional (1D) crystals formed has been achieved within single walled carbon nanotubes (SWNTs). These species have been imaged directly or indirectly by the techniques of conventional or restored high-resolution transmission electron microscopy (HRTEM). Here we present evidence for the formation of continuous semiconducting 1D nanowires formed within single wall carbon nanotubes [1]. In addition to this, we were able to determine the 3D structure of the average crystal structure formed within SWNTs of approximately equal diameter from a pair of restored images obtained from two encapsulated HgTe 1D crystals related by tilt and a rotation symmetry operations. The SWNTs were filled via a capillary wetting technique [2] with the semi-metal HgTe. The resulting HgTe@SWNT composite was analysed (see Fig. 1(a)) using a restored HRTEM technique approach to that used to image a $3 \times 3 \mathrm{KI}$ crystal formed within a $1.6 \mathrm{~nm}$ SWNT [3].

We were able to produce numerous restored phase images of $1 \mathrm{D}$ HgTe crystals such as those reproduced in Figure 1(a) I and II. These images were produced from focal series recorded on a JEOL 3000F microscope equipped with a Gatan 1024x1024 CCD camera using a previously described procedure [3,4]. At first sight the microstructures reproduced in Fig. 1(a) appear unrelated but due to the ability to measure the relative focal 'heights' of the restored sub-regions of each of the two images [4], it was possible to perform a comparative analysis of both images in order to establish the link between both of the encapsulated fragments. The plots in Figure (b) show how the tilt angles of the tubes in (a) vary between 0 for $\mathbf{I}$ and $-26^{\circ}$ for II while their detailed microstructures enabled the fabrication of various candidate structure models (two of which are shown in Figure 1(c)) comprising various arrangements of $\mathrm{Hg}_{2} \mathrm{Te}_{2}$ layers which formed a motif common to both microstructures. By varying the Te-Hg-Te bond angle $(\gamma)$, the relative tilt angle of the SWNT with respect to the electron beam $(\gamma)$ and also the respective angle of orientation of successive alternating $\mathrm{Hg}_{2} \mathrm{Te}_{2}$ layers $(\phi)$ with respect to each other, it was possible to produced simulation maps (e.g. Figure 1(d)), to successfully cross correlate the two images with sets of simulations and thereby successfully establish the structural relationship between the two crystal fragments. The net result is that the two details reproduced from the original image in Figure 1(e) can be related by two symmetry operations (i.e. a tilt and a rotation) and these can successfully be simulated (cf. Fig. 1(e) and (f)).

\section{References}

[1]R. Carter, J. Sloan, A.I. Kirkland, R. Meyer, P. Lindan, A. Vlandas, J.L. Hutchison (submitted) [2]J. Sloan, D. M. Wright, H. G. Woo, S. R. Bailey, G. Brown, A. P. E. York, K. S. Coleman, J. L. Hutchison and M. L. H. Green, Chem. Commun. (1999) 699.

[3]R. R. Meyer, J. Sloan, R. E. Dunin-Borowski, A. I. Kirkland, M. C. Novotny, S. R. Bailey, J. L. Hutchison and M. L. H. Green, Science 289 (2000) 1324.

[4] S. Friedrichs, J. Sloan, J.L. Hutchison, M.L.H. Green, R.R. Meyer and A.I. Kirkland, Phys. Rev. B, 64 (2001) 045406. 
(a)

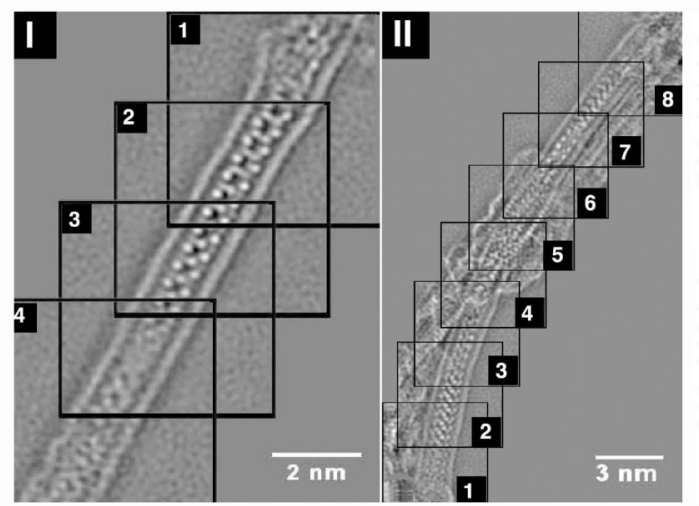

(d)
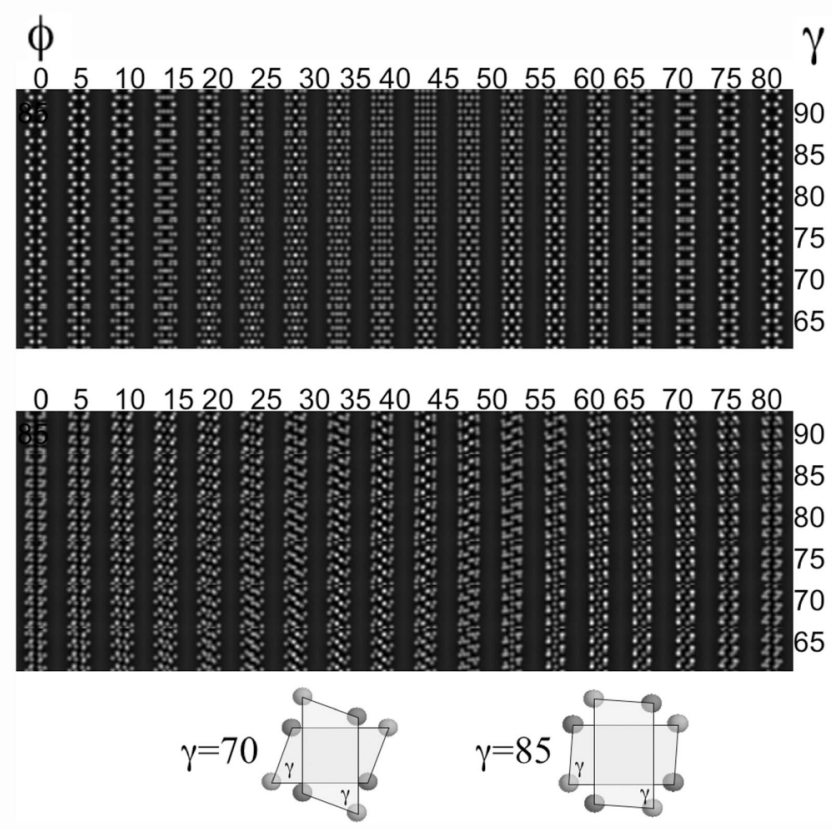

(b)
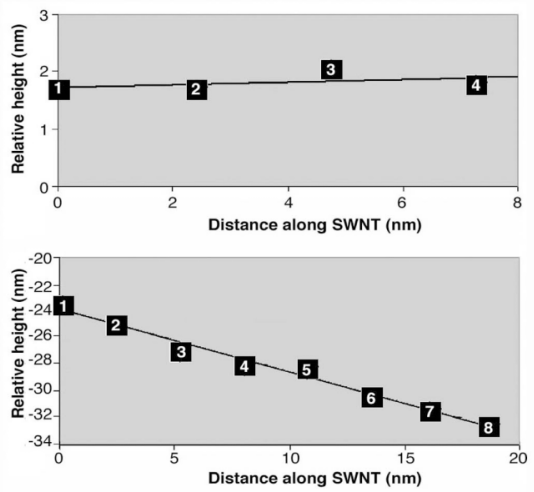

(e)

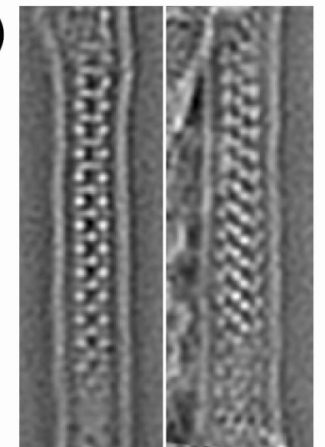

(f)

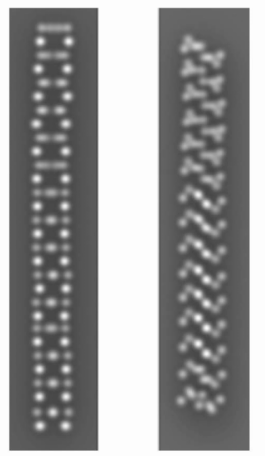

(c)

sample candidate I

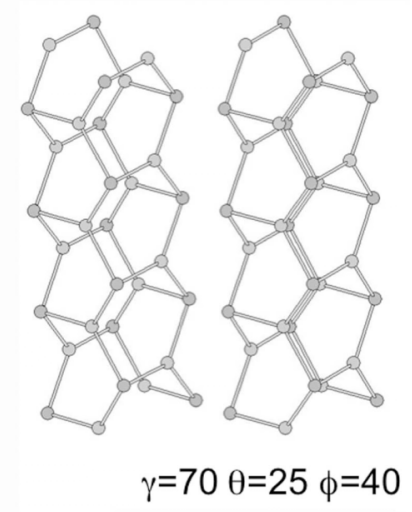

sample candidate II

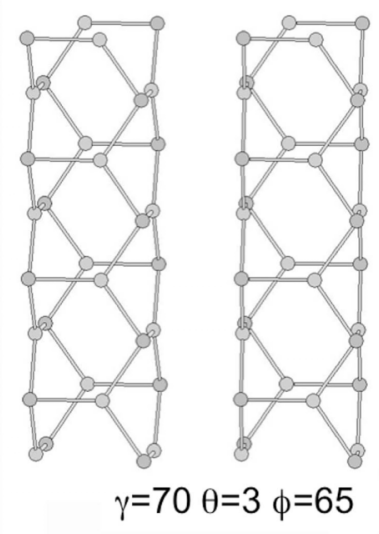

Figure 1(a) Two super-resolved HRTEM composite images (I and II) of 1D crystals of $\mathrm{HgTe}$ encapsulated within two separate nanotubes with restored sub regions indicated. (b) Relative heights of the subregions indicated in (a) plotted versus the distance along the SWNT (top and bottom plots correspond to composite images I and II in (a), respectively. (c) Two candidate structural models with $\mathrm{Hg}_{2} \mathrm{Te}_{2}$ motifs in which the Te-Hg-Te bond angle (i.e. $\gamma$ ), the relative tilt angle of the 1D fragment (i.e. $\theta$ ) and the angle of orientation of alternating $\mathrm{Hg}_{2} \mathrm{Te}_{2}$ layers (i.e. $\phi$ ) is varied. (d) Phase image simulation plots for which $\theta$ is held constant but for which $\gamma$ is varied with respect to $\phi$. (e) enlarged details from (a) I (left) and II (right). (f) 'Best fit' composite simulations based on two models related by a $26^{\circ}$ tilt and a ca. $20^{\circ}$ rotation. Systematic twists were also introduced into both models. 